\title{
Experimental observation of chimeras in coupled-map lattices
}

\author{
Aaron M. Hagerstrom ${ }^{1,2 \star}$, Thomas E. Murphy ${ }^{1,3}$, Rajarshi Roy ${ }^{1,2,4}$, Philipp Hövel ${ }^{5,6}$, \\ Iryna Omelchenko ${ }^{5,6}$ and Eckehard Schöll ${ }^{5}$
}

\begin{abstract}
Networks of nonlocally coupled phase oscillators ${ }^{1}$ can support chimera states in which identical oscillators evolve into distinct groups that exhibit coexisting synchronous and incoherent behaviours despite homogeneous coupling ${ }^{2-6}$. Similar nonlocal coupling topologies implemented in networks of chaotic iterated maps also yield dynamical states exhibiting coexisting spatial domains of coherence and incoherence ${ }^{7,8}$. In these discrete-time systems, the phase is not a continuous variable, so these states are generalized chimeras with respect to a broader notion of incoherence. Chimeras continue to be the subject of intense theoretical investigation, but have yet to be realized experimentally ${ }^{6,9-16}$. Here we show that these chimeras can be realized in experiments using a liquid-crystal spatial light modulator to achieve optical nonlinearity in a spatially extended iterated map system. We study the coherenceincoherence transition that gives rise to these chimera states through experiment, theory and simulation.
\end{abstract}

Our system is an experimental realization of a coupled-map lattice (CML), a class of systems that has received sustained theoretical interest for the past three decades. Although the dynamics and statistical physics of CML systems have been theoretically explored, very few (if any) experimental realizations exist $^{17-22}$. In our experiments, we create CML dynamics by using a liquid-crystal spatial light modulator (SLM) to control the polarization properties of an optical wavefront. We may electronically introduce any desired coupling topology including nearest neighbour, nonlocal, small world and scale free. In this work, we impose periodic boundary conditions for both onedimensional (1D) and 2D nonlocally coupled maps. Thus, we have developed a powerful experimental technique to observe the parallel evolution of the dynamics of arrays of coupled maps numbering up to thousands or more depending on the goals of the experiment.

Figure 1 shows the experimental set-up of the optical CML. Polarization optics create a nonlinear relationship between the spatially dependent phase shift applied by the SLM and the intensity of the light falling on the camera: $I(\phi)=(1-\cos (\phi)) / 2$. The operation of the experimental apparatus is described in the Methods. Both the SLM and the camera frames are partitioned into an $M \times M$ array of square regions. These regions correspond to nodes in the network of coupled maps. Time evolution of the network is achieved by iteratively updating the phase applied by each region of the SLM in a way that depends on the intensity measured by the camera.

We present results for two different coupling schemes shown schematically in Fig. 1b,c. In the 1D configuration, the elements in the array are arranged as a ring with periodic boundary conditions.

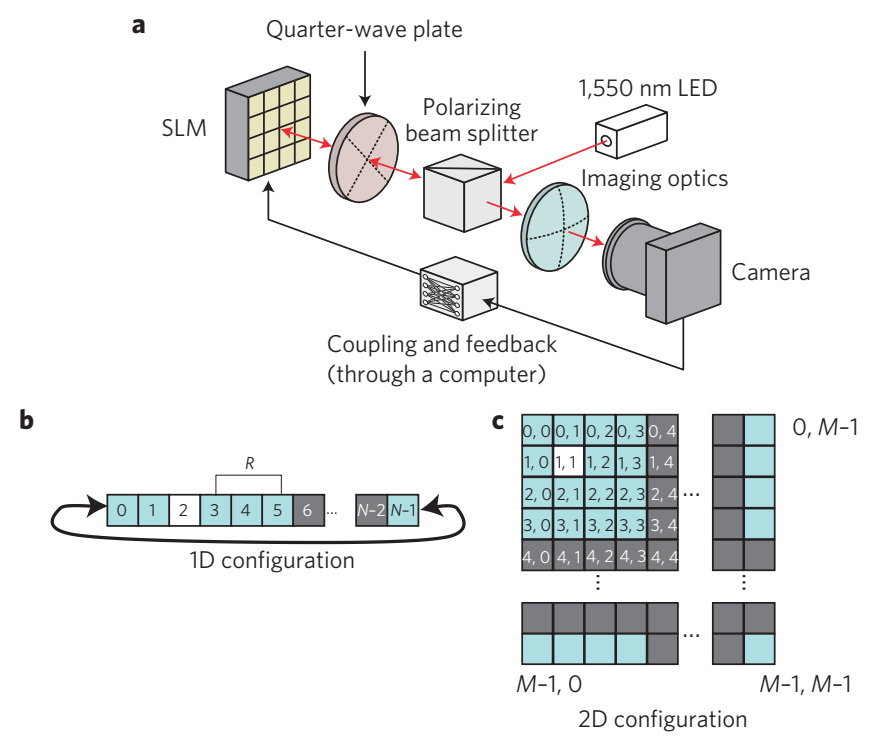

Figure 1 | Experimental apparatus. a, Optical configuration. Polarization optics create a nonlinear relationship between the spatially dependent phase shift applied by the SLM and the intensity of the light falling on the camera. Feedback and coupling are implemented using a computer. $\mathbf{b}, \mathbf{c}$, Schematics of the 1D (b) and 2D (c) coupling configurations are shown. The site highlighted in white is updated based on the sites indicated in blue. As the elements are coupled diffusively to their neighbours within a range $R$ in either one or two dimensions with periodic boundary conditions, the coupling is identical for all oscillators.

The SLM is treated as a $1 \mathrm{D}$ lattice with the elements coupled in a raster-ordered arrangement. If $\phi_{i}^{n}$ is the phase of the $i$ th element in the ring at the $n$th iteration, and $I\left(\phi_{i}^{n}\right)$ is the intensity measured by the corresponding region in the camera, then the phase is updated according to

$$
\phi_{i}^{n+1}=2 \pi a\left\{I\left(\phi_{i}^{n}\right)+\frac{\epsilon}{2 R} \sum_{j=-R}^{R}\left[I\left(\phi_{i+j}^{n}\right)-I\left(\phi_{i}^{n}\right)\right]\right\}
$$

where $i=0, \ldots, N-1$ in the $1 \mathrm{D}$ ring configuration and the index $i$ is periodic modulo $N$. Thus, each element is coupled diffusively to all of the elements within a distance $R$ on either side, and $\epsilon$ describes the strength of the coupling. The parameter $a$ controls the temporal dynamics of an isolated map. We choose $a=0.85$

\footnotetext{
${ }^{1}$ Institute for Research in Electronics and Applied Physics, University of Maryland, College Park, Maryland 20742, USA, ${ }^{2}$ Department of Physics, University of Maryland, College Park, Maryland 20742, USA, ${ }^{3}$ Department of Electrical and Computer Engineering, University of Maryland, College Park, Maryland 20742, USA, ${ }^{4}$ Institute for Physical Science and Technology, University of Maryland, College Park, Maryland 20742, USA, ${ }^{5}$ Institut für Theoretische Physik, Technische Universität Berlin, Hardenbergstraße 36, 10623 Berlin, Germany, ${ }^{6}$ Bernstein Center for Computational Neuroscience, Humboldt-Universität zu Berlin, Philippstraße 13, 10115 Berlin, Germany. *e-mail: aaron.hagerstrom@gmail.com.
} 
a

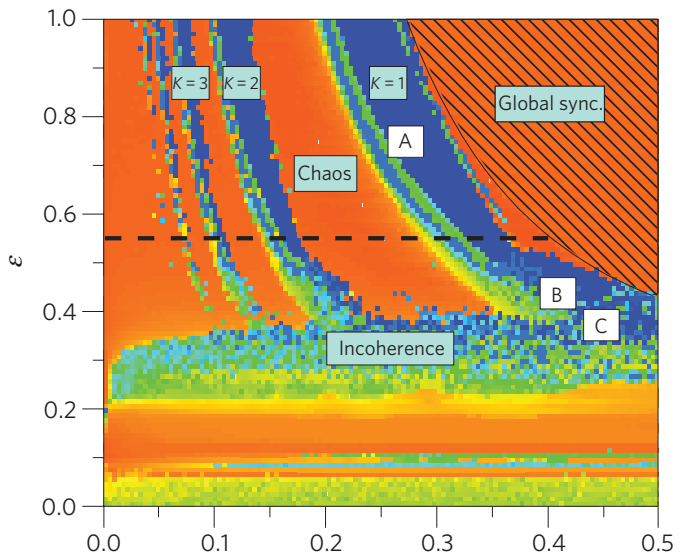

c
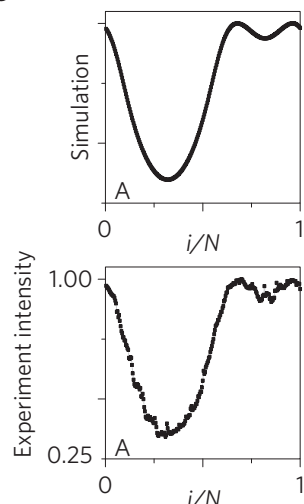

$i / N$
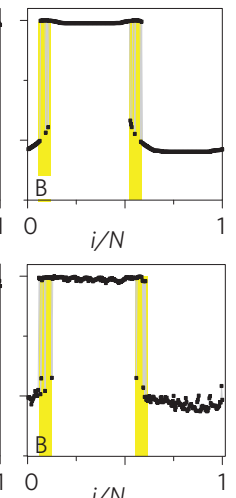

b

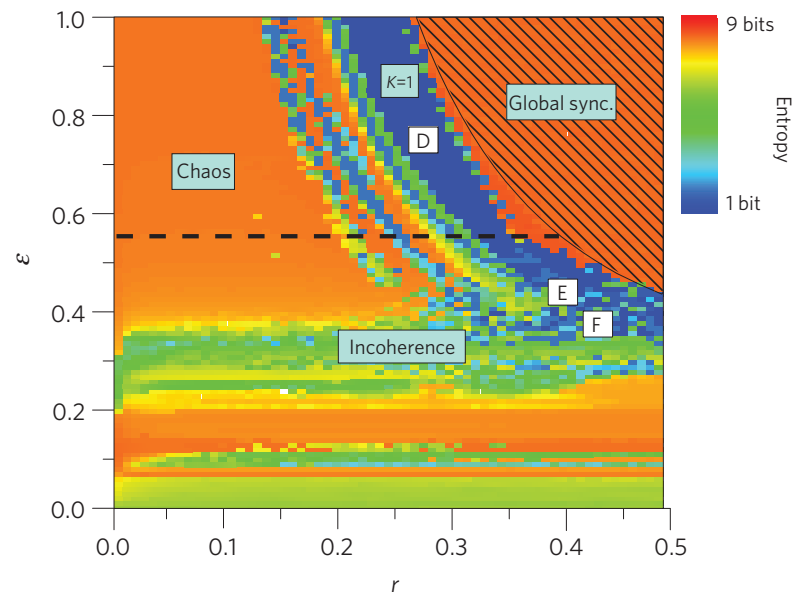

d
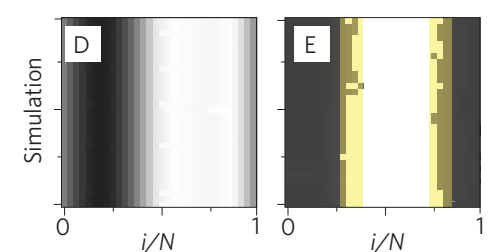

i/N

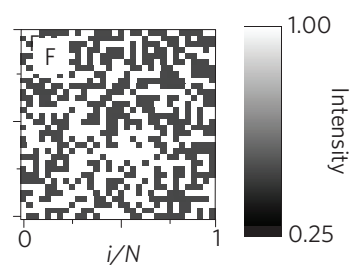

i/N

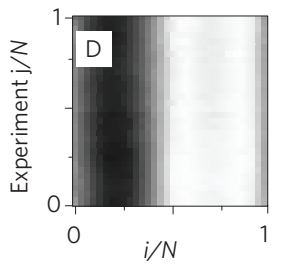

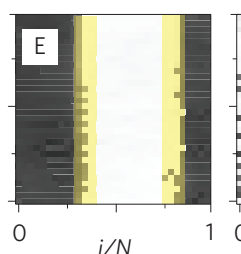

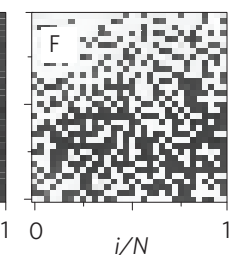

Figure 2 | Parameter space of the 1D and 2D CML systems. a, Parameter space of the 1D configuration ( $N=256$ elements). Blue corresponds to low entropy and periodicity; orange corresponds to high entropy and chaos. The dashed line indicates the critical coupling strength $\epsilon_{\mathrm{c}}=0.54$. There is a series of tongues containing profiles which are periodic in time and have spatial wavenumbers $K=1, K=2$ and $K=3$ as indicated. $\mathbf{b}$, Parameter space of the $2 \mathrm{D}$ configuration ( $128 \times 128$ lattice). There is a prominent $K=1$ tongue, and two tongues with more complex spatial patterns that are not characterized by a wavenumber. c, Experimental and numerical realizations of the 1D system. In B, the incoherent region is highlighted in yellow. Labels A (coherence), B (chimera) and $C$ (incoherence) show positions in the parameter space of $\mathbf{a}$. $\mathbf{d}$, Experimental and numerical realizations of the $2 \mathrm{D}$ system. In $\mathrm{E}$, the incoherent region is highlighted.

such that the local map $\phi^{n+1}=f\left(\phi^{n}\right) \equiv 2 \pi a I\left(\phi^{n}\right)$ is chaotic with a Lyapunov exponent $\lambda \approx 0.58$.

We also examine a $2 \mathrm{D}$ coupling scheme. In this configuration, each element is coupled to its neighbours within a square region, and the boundary conditions are periodic in both dimensions.

$$
\phi_{i, j}^{n+1}=2 \pi a\left\{I\left(\phi_{i, j}^{n}\right)+\frac{\epsilon}{4 R^{2}} \sum_{k, l=-R}^{R}\left[I\left(\phi_{i+k, j+l}^{n}\right)-I\left(\phi_{i, j}^{n}\right)\right]\right\}
$$

Figure 2a,b characterizes the dependence of the dynamics of the $1 \mathrm{D}$ and the $2 \mathrm{D}$ systems on coupling strength and range by plotting a measure of temporal entropy (see Methods). The colour maps were obtained through numerical simulation of equations (1) and (2). A period-2 profile will have an entropy of 1 bit, and a chaotic profile will have an entropy close to 9 bits, because 512 bins were used. We see spatial profiles that are periodic in time, as well as profiles that are chaotic in time. There is a rich variety of both coherent and incoherent spatial structures. The thin vertical regions marked $K=1,2,3$ in Fig. $2 \mathrm{a}$ indicate conditions for which the dynamical state is periodic in time and forms a spatially periodic standing-wave pattern with a spatial wavenumber of $K$. Within each of these regions, there is a transition from spatial coherence to incoherence with decreasing coupling strength $\epsilon$, as predicted in refs 7,8 . Equations (1) and (2) admit a globally synchronous solution. The hatched region in Fig. 2a,b indicates the region in parameter space for which global synchrony is stable, which is identical between the 1D and 2D systems.

A comparison of the $1 \mathrm{D}$ and $2 \mathrm{D}$ systems reveals that these systems show equivalent behaviour within a limited region in parameter space. In the $2 \mathrm{D}$ system there is a prominent region marked $K=1$, which contains profiles that are homogeneous in one direction. Every solution of the $1 \mathrm{D}$ problem corresponds to a solution of the $2 \mathrm{D}$ problem that is uniform in one direction, that is, $\phi_{i, j}=\phi_{i}$. Although $K=2,3 \ldots$ profiles also exist in principle, only the $K=1$ profile is observed in simulation and experiment in the $2 \mathrm{D}$ system within the evolution time we consider. Simulations also reveal two smaller regions that contain complex profiles with spatial structures that cannot be realized in one dimension.

Figure $2 \mathrm{c}$ shows experimental and numerical realizations of three profiles from the $K=1$ region. The sequence A, B, C shows a progression from spatial coherence to spatial incoherence. All profiles have a temporal period of 2 . At a coupling radius $r \equiv R / N=$ $70 / 256 \approx 0.27$ and $\epsilon=0.75(\mathrm{~A})$, the profile has a smooth spatial variation. At $r=105 / 256 \approx 0.41$ and $\epsilon=0.44(\mathrm{~B})$, the profile shows two large domains of coherent, synchronized behaviour separated by narrow but finite regions of incoherent behaviour highlighted in yellow. Numerical evidence indicates that the width of these regions as a fraction of the system size remains constant as $N$ increases, and hence this is not a boundary effect (see Supplementary Information). Although the entire profile is periodic in time, the 
a

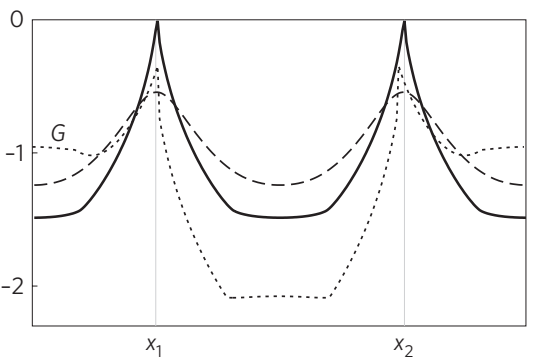

b

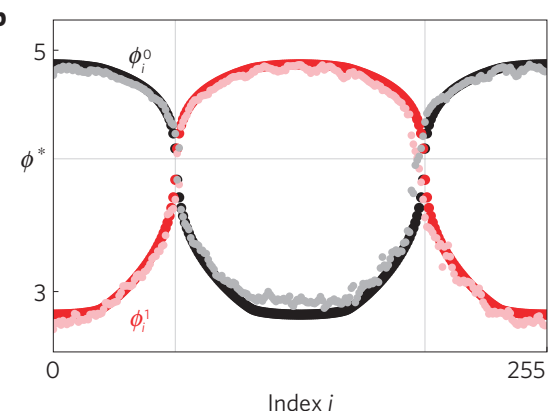

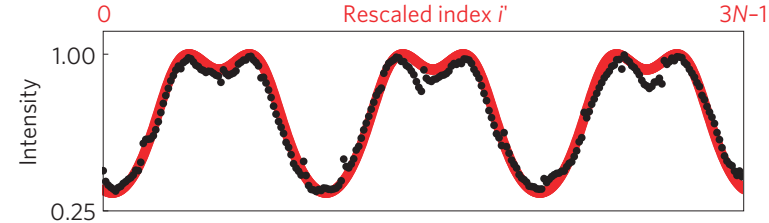

d

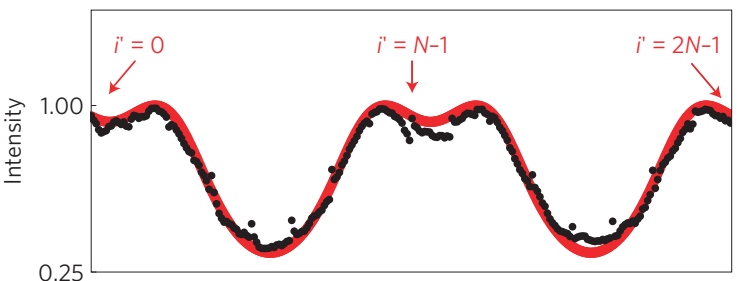

e

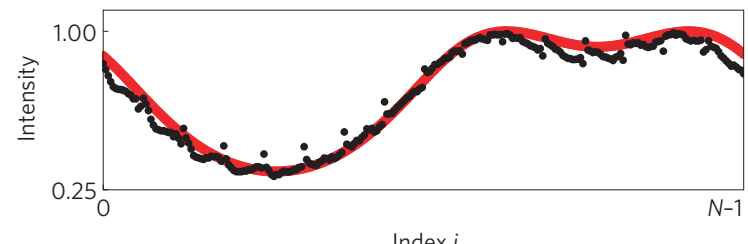

Figure 3 | Critical coupling and scaling of coherent profiles. a, Condition $G$ for breaking of the smooth profile. The solid curve corresponds to the critical coupling strength $\epsilon_{\mathrm{c}}=0.54$. The dashed and dotted curves refer to $\epsilon=0.675>\epsilon_{\mathrm{c}}$ and $\epsilon=0.475<\epsilon_{\mathrm{c}}$, respectively. $\mathbf{b}$, Snapshots of the period- 2 solution for even $\left(\phi_{i}^{0}\right.$ (black)) and odd $\left(\phi_{i}^{1}\right.$ (red)) time steps at the critical coupling strength of $\mathbf{a}$. The light red and grey dots refer to the experiment. Points $x_{1}$ and $x_{2}$ are discontinuity points, where the profile breaks, and $\phi^{*}$ marks the fixed point of the local map. Other parameters: $N=256, a=0.85$ and $r=88 / 256 \approx 0.34$. c-e, Scaling of coherent intensity profiles. The measured coherent profiles with spatial periods $K=1,2,3$ are shown in black for coupling radius $r_{3}=22 / 256 \approx 0.09(\mathbf{c}), r_{2}=33 / 256 \approx 0.13(\mathbf{d})$ and $r_{1}=66 / 256 \approx 0.26$ (e). The profile $K=1$ numerically obtained from equations $(1)$ and (5) and its rescaled profile are shown in red in $\mathbf{c}$ and $\mathbf{d}, \mathbf{e}$, respectively. Other parameters: $a=0.85, \epsilon=0.8$ and $N=256$.

dynamics of the coherent and incoherent regions are qualitatively different in their degree of spatial coherence: within the incoherent intervals there are numerous admissible combinations of upper and lower states whose multiplicity scales exponentially with the system $\operatorname{size}^{7}$. This mixture of qualitatively different behaviours is analogous to the chimera states discussed in refs $2-4,9-11$, where an array of identical oscillators splits into two domains: one coherent and phase locked, the other incoherent and desynchronized. Unlike the chimeras in continuous-time phase oscillators ${ }^{2,3}$, the generalized chimeras here and in refs 7,8 have two coherent parts (with high and low intensity, respectively) and two incoherent parts, as a result of their mechanism of nascence from the completely coherent $K=1$ spatial profile. Finally, at $r=115 / 256 \approx 0.45$ and $\epsilon=0.375$ (C), the profile is completely incoherent. The same scenario occurs in the $K=2$ and $K=3$ regions and can be interpreted through a spatial rescaling described below.

Figure $2 \mathrm{~d}$ shows snapshots of experimental and numerical realizations of the $2 \mathrm{D}$ system in a $32 \times 32$ lattice, obtained with similar parameter values to those used in Fig. $2 \mathrm{~b}$. All of these realizations are periodic in time with period 2, except $(\mathrm{E})$, which has a period of 4 in the experimental realization shown. As for the 1D system, the $2 \mathrm{D}$ system undergoes a coherence-incoherence transition as $\epsilon$ is decreased. At $r=9 / 32 \approx 0.28$ and $\epsilon=0.75$, the system exhibits a smooth profile (D). For $r=13 / 32 \approx 0.41$ and $\epsilon=0.44$, there is a chimera-like coexistence of coherence and incoherence (E). Finally, at $r=14 / 32 \approx 0.44$ and $\epsilon=0.375$, the system is fully incoherent $(\mathrm{F})$.

The transition from coherence to incoherence can be explained analytically. We derive the critical coupling strength $\epsilon_{\mathrm{c}}$ at which the smooth profile breaks up giving rise to the incoherent regions (B) of Fig. 2c. Considering the spatially continuous version of equation (1) and solutions with wavenumber $K=1$ and period-2 dynamics in time, the system dynamics are given by alternating profiles $\phi^{0}(x)$ and $\phi^{1}(x)$ for even and odd time steps, respectively. By evaluating the spatial derivative of this equation with the profiles $\phi^{0}(x)$ and $\phi^{1}(x)$ at the positions where the smooth profiles break up, we obtain two equations that, when multiplied, yield the condition (see Supplementary Information)

$$
1=(2 \pi a)^{2}(1-\epsilon)^{2} \partial_{x} I\left(\phi^{0}(x)\right) \partial_{x} I\left(\phi^{1}(x)\right)
$$

Taking into account the local dynamics, we define the following function as a deviation from equation (3)

$$
G(x)=(\pi a)^{2}(1-\epsilon)^{2} \sin \left(\phi^{0}(x)\right) \sin \left(\phi^{1}(x)\right)-1
$$

where we can use numerically obtained profiles $\phi^{0}(x)$ and $\phi^{1}(x)$ for given coupling parameters $r$ and $\epsilon$.

The critical coupling strength $\epsilon_{\mathrm{c}}$ at which coherence is broken can be determined by applying the condition $G(x)=0$ at the crossing points $x_{1}$ and $x_{2}$ where $\phi^{0}(x)=\phi^{1}(x) \equiv \phi^{*}$ as shown in Fig. 3b. Applying this condition to equation (1) yields

$$
\epsilon_{\mathrm{c}}=1-\frac{1}{(\pi a)\left|\sin \phi^{*}\right|}
$$

The 1D local map $f$ has three fixed points for $a=0.85: \phi_{0}^{*}=0$, $\phi_{1}^{*} \approx 0.79$ and $\phi_{2}^{*} \approx 4.13$. The fixed point $\phi_{2}^{*}$ plays the role of a saddle (unstable fixed point), which separates the attractor basins of the two stable fixed points of the twice-iterated local map, which correspond to the period-2 solution of the local map (see Supplementary Fig. S5). Approximating $\phi^{*}$ by $\phi_{2}^{*}$ in equation (4) we obtain $\epsilon_{\mathrm{c}} \approx 0.55$, which is close to the numerical result $\epsilon_{\mathrm{c}}=$ 0.54 shown in Fig. 3a,b.

One can further obtain an approximate full analytical solution (for $a>0.6$ ) by Taylor expanding the local map $f$ about $\pi$ using $\cos \phi^{*} \approx-1+1 / 2 \psi^{2}$, where $\psi=\phi^{*}-\pi$. This yields an equation for the fixed point $\pi+\psi \approx \pi a\left(2-1 / 2 \psi^{2}\right)$, and we find $\psi \approx\left(-1+\sqrt{1+2 \pi^{2} a(2 a-1)}\right) /(\pi a)$.

This gives $\psi \approx 0.96, \phi^{*} \approx 4.10$, and through equation (4) $\epsilon_{\mathrm{c}}=0.54$. Figure 3a shows the function $G$ for $\epsilon_{\mathrm{c}}=0.54$ (solid line), $\epsilon=0.675$ (dashed) and $\epsilon=0.475$ (dotted). Figure $3 \mathrm{~b}$ depicts the 
corresponding snapshots $\phi_{i}^{0}$ and $\phi_{i}^{1}$ for $\epsilon_{\mathrm{c}}=0.54$. The profiles $\phi_{i}^{0}$ and $\phi_{i}^{1}$ cross at $\phi^{*} \approx 4.10$. The experimental results are added as light red and grey dots in Fig. $3 \mathrm{~b}$.

In addition we analytically demonstrate the invariance of the coherent profiles of different wavenumbers for equation (1) by a scaling relation (Methods). Figure 3c-e shows the scaling of profiles with $K=1,2,3$ obtained from the experimental realization with $N=256$ for coupling ranges $R_{3}=22$ (Fig. 3c), $R_{2}=33$ (Fig. $3 \mathrm{~d}$ ) and $R_{1}=66$ (Fig. $3 \mathrm{e}$ ) as black dots. The numerical profile from equation (1) for $K=1$ and the rescaled profiles are depicted in red in Fig. 3c,d,e, respectively.

In summary we have constructed a versatile experimental system to explore the spatio-temporal dynamics of arbitrary networks of coupled maps. The nodes in the network are nonlocally and homogeneously coupled, and we observe the formation of coexisting spatially coherent and incoherent domains as the coupling parameters are varied. This behaviour is observed in both $1 \mathrm{D}$ and 2D systems.

\section{Methods}

Experimental apparatus. Figure 1 shows the experimental set-up of the optical CML. The SLM (Boulder Nonlinear Systems P512-1550), with an active area of $7.68 \times 7.68 \mathrm{~mm}^{2}$ and $512 \times 512$ pixels, is illuminated by collimated $1,550 \mathrm{~nm}$ light from a fibre-coupled superluminescent diode. The light passes through a polarizing beam splitter and a quarter-wave plate oriented at a $45^{\circ}$ angle to the direction of linear polarization of the incident light, is reflected by the SLM with a relative phase shift between the fast and slow axes, and passes again through the quarter-wave plate and the beam splitter. We use the computer-controlled SLM to apply an arbitrary spatially dependent phase modulation to the optical wavefront using a birefringent liquid crystal sandwiched between an array of reflective electrodes and a transparent cover glass. Each of the electrodes acts as an independent pixel that can impose an arbitrary phase shift from 0 to $2 \pi$ between the two polarization components of the incoming light by applying an electric field to reorient the liquid crystals $^{23}$. The polarization optics create a nonlinear relationship between the phase shift applied by the SLM and the intensity of light falling on the camera (Goodrich SU320KTSW-1.7RT/RS170), with an active area of $8 \times 6.4 \mathrm{~mm}^{2}$ and $320 \times 256$ pixels. A selected square area of $256 \times 256$ pixels was used. The phase shift $\phi_{i, j}$ and intensity $I$ for a given pixel $(i, j)$ are related by

$$
I\left(\phi_{i, j}\right)=\frac{1}{2}\left(1-\cos \phi_{i, j}\right), \quad i, j=0, \ldots, M-1
$$

The intensity has been normalized to be between 0 and 1 . A lens is used to project an image of the SLM onto an infrared camera, which records the 2D intensity pattern $I\left(\phi_{i, j}\right)$. We construct a network of iterated maps by using the computer to communicate between the camera and the SLM. Both the SLM and the camera screens are partitioned into an $M \times M$ array of square regions. These regions correspond to nodes in the network of coupled maps. Feedback is achieved by iteratively updating the phase applied by each region on the SLM in a way that depends on the intensity measured by the camera.

Entropy calculation. In Fig. 2a,b, the colour of each point corresponds to a single numerical simulation. Lattice sizes of 256 for the $1 \mathrm{D}$ case and $128 \times 128$ for the $2 \mathrm{D}$ case were used. Initially, the phase of each node is random and uniformly distributed between 0 and $2 \pi$. In each simulation we discard 50,000 transient iterations. Using the next 5,000 iterations, we construct one histogram for each of the $N$ nodes in the network, binning the values the phase achieves in these iterations. For each lattice site, 512 bins were used. Thus, we estimate $p_{i}^{n}$, the fraction of the time that node $i$ will spend in the $n$th bin. From these statistics we obtain the entropy, which is then averaged over the $N$ nodes in the system

$$
H=-\frac{1}{N} \sum_{i=0}^{N-1} \sum_{n=0}^{511} p_{i}^{n} \log _{2} p_{i}^{n}
$$

We note that for this system the temporal behaviour of the whole network is either chaotic or periodic, and the spatial average of the entropy will not differ substantially from the entropy of any given node. We also note that this calculation characterizes only the temporal behaviour of the system, and does not distinguish between coherence and incoherence.

Scaling of coherent profiles. As a second analytic finding we demonstrate the scaling relation for coherent profiles of different wavenumbers. Considering the spatially continuous version of the 1D system in equation (1) (see Supplementary Information),

$$
\phi_{K^{\prime}}^{n+1}(x)=f\left(\phi_{K^{\prime}}^{n}(x)\right)+\frac{\epsilon}{2 r} \int_{x-r}^{x+r}\left[f\left(\phi_{K^{\prime}}^{n}(y)\right)-f\left(\phi_{K^{\prime}}^{n}(x)\right)\right] \mathrm{d} y
$$

where the coupling strength $\epsilon$ is fixed, and $f(\phi)=2 \pi a I(\phi)$, we assume that there exist two solutions $\phi_{K^{\prime}}(x)$ and $\phi_{K}(x)$ with spatial periods $K^{\prime}$ and $K$, respectively. It follows that the dynamics of $\phi_{K^{\prime}}(x)$ is identical to the dynamics of $\phi_{K}(\tilde{x})$ at a rescaled spatial position $\tilde{x}=\left(x K^{\prime}\right) / K$ for a correspondingly rescaled coupling radius $\tilde{r}=\left(r K^{\prime}\right) / K$. This demonstrates the invariance of the solutions $\phi_{K}$ for appropriate rescaling of the coupling range (see Supplementary Information).

\section{Received 19 March 2012; accepted 14 June 2012; published online 15 July 2012}

\section{References}

1. Kuramoto, Y. Chemical Oscillations, Waves and Turbulence (Springer, 1984).

2. Kuramoto, Y. \& Battogtokh, D. Coexistence of coherence and incoherence in nonlocally coupled phase oscillators. Nonlinear Phen. Complex Syst. 5, 380-385 (2002).

3. Abrams, D. M. \& Strogatz, S. H. Chimera states for coupled oscillators. Phys. Rev. Lett. 93, 174102 (2004).

4. Abrams, D. M., Mirollo, R., Strogatz, S. H. \& Wiley, D. A. Solvable model for chimera states of coupled oscillators. Phys. Rev. Lett. 101, 084103 (2008).

5. Martens, E. A., Laing, C. R. \& Strogatz, S. H. Solvable model of spiral wave chimeras. Phys. Rev. Lett. 104, 044101 (2010).

6. Motter, A. E. Nonlinear dynamics: Spontaneous synchrony breaking. Nature Phys. 6, 164-165 (2010).

7. Omelchenko, I., Maistrenko, Y. L., Hövel, P. \& Schöll, E. Loss of coherence in dynamical networks: Spatial chaos and chimera states. Phys. Rev. Lett. 106, 234102 (2011).

8. Omelchenko, I., Riemenschneider, B., Hövel, P., Maistrenko, Y. L. \& Schöll, E. Transition from spatial coherence to incoherence in coupled chaotic systems. Phys. Rev. E 85, 026212 (2012).

9. Laing, C. R. The dynamics of chimera states in heterogeneous Kuramoto networks. Physica D 238, 1569-1588 (2009).

10. Laing, C. R. Chimeras in networks of planar oscillators. Phys. Rev. E 81, 066221 (2010).

11. Laing, C. R. Fronts and bumps in spatially extended Kuramoto networks. Physica D 240, 1960-1971 (2011).

12. Martens, E. A. Bistable chimera attractors on a triangular network of oscillator populations. Phys. Rev. E 82, 016216 (2010).

13. Martens, E. A. Chimeras in a network of three oscillator populations with varying network topology. Chaos 20, 043122 (2010).

14. Wolfrum, M. \& Omel'chenko, O. E. Chimera states are chaotic transients. Phys. Rev. E 84, 015201 (2011)

15. Sethia, G. C., Sen, A. \& Atay, F. M. Clustered chimera states in delay-coupled oscillator systems. Phys. Rev. Lett. 100, 144102 (2008).

16. Sen, A., Dodla, R., Johnston, G. L. \& Sethia, G. C. in Understanding Complex Systems (ed. Atay, F. M.) 1-41 (Springer, 2010).

17. Waller, I. \& Kapral, R. Spatial and temporal structure in systems of coupled nonlinear oscillators. Phys. Rev. A 30, 2047-2055 (1984).

18. Kaneko, K. Overview of coupled map lattices. Chaos 2, 279-282 (1992).

19. Kaneko, K. Theory and Applications of Coupled Map Lattices (John Wiley, 1993).

20. Kaneko, K. Period-doubling of kink-antikink patterns, quasiperiodicity in anti-ferro-like structures and spatial intermittency in coupled logistic lattice. Prog. Theor. Phys. 72, 480-486 (1984).

21. Pikovsky, A. S., Rosenblum, M. \& Kurths, J. Synchronization: A Universal Concept in Nonlinear Sciences Vol. 12 (Cambridge Univ. Press, 2003).

22. Boccaletti, S., Kurths, J., Osipov, G., Valladares, D. L. \& Zhou, C. S. The synchronization of chaotic systems. Phys. Rep. 366, 1-101 (2002).

23. Khoo, I. C. Liquid Crystals Vol. 64 (John Wiley, 2007).

\section{Acknowledgements}

We thank X. Li for her help in developing the early stages of the experiment. A.M.H., T.E.M. and R.R. acknowledge support by DOD MURI Grant No. ONR N000140710734. P.H. and I.O. acknowledge support by the BMBF under grant no. 01GQ1001B (Förderkennzeichen). E.S. acknowledges support by Deutsche Forschungsgemeinschaft in the framework of SFB910.

\section{Author contributions}

A.M.H. developed the experimental system under the guidance of T.E.M. and R.R. and performed measurements and numerical simulations to characterize the parameter space of the 1D and 2D systems. P.H., I.O. and E.S. conducted theoretical and numerical studies of the scaling relation and critical coupling strength. All authors discussed the results and wrote the manuscript.

\section{Additional information}

The authors declare no competing financial interests. Supplementary information accompanies this paper on www.nature.com/naturephysics. Reprints and permissions information is available online at www.nature.com/reprints. Correspondence and requests for materials should be addressed to A.M.H. 